\title{
From the Editors' Desk: The July Effect
}

\author{
Richard L. Kravitz, MD, MSPH' , Mitchell D. Feldman, MD, MPhiP , and G. Caleb Alexander, MD, $M S^{3}$ \\ 'Division of General Medicine, University of California at Davis, Sacramento, CA, USA; ${ }^{2}$ Division of General Internal Medicine, University of \\ California, San Francisco, San Francisco, CA, USA; ${ }^{3}$ Division of General Internal Medicine, University of Chicago, Chicago, IL, USA.
}

\author{
J Gen Intern Med 26(7):677 \\ DOI: $10.1007 / \mathrm{s} 11606-011-1736-3$ \\ (C) Society of General Internal Medicine 2011
}

$\mathrm{W}$ hile the heat outside may be stifling, at America's teaching hospitals, July brings a waft of fresh air. Senior residents have graduated, new interns have arrived. The wards are abuzz with excitement. We know colleagues who ask for ward duty assignments in July, just for the thrill of it. "The interns hang on your every word," they say. "It's a good feeling."

This July brings a surfeit of good feelings, but there will be challenges as well. New residency work hour rules proposed by the Accreditation Council for Graduate Medical Education (ACGME) in 2010 are due to take effect this month. The rules call for increased supervision of physicians-in-training, tighter limits on moonlighting, and reduced work hours for first-year residents. With interns limited to shifts of 16 hours, residency programs have been scrambling to create new call schedules and systems for cross-coverage. No one really knows what will happen. By imposing uniform work hours on a national basis, the ACGIM has impeded our ability to learn about which specific standards result in better outcomes. As SGIM member Kevin Volpp and colleagues pointed out in the Annals of Internal Medicine late last year, "The main disadvantage of the [regulatory] approach is that there is no ability to determine whether different standards would have worked better to reduce resident fatigue while improving patient safety."

Fortunately, other initiatives to improve the quality and safety of patient care are undergoing more careful scientific scrutiny, many in the pages of this journal. For example, in this issue Simon et al. show that online messaging improves patient followup for depression compared with usual care. While prior randomized clinical trials have clearly demonstrated that care management programs improve clinical outcomes in depression, this study may well be the first that uses online messaging to deliver follow up care to patients initiating antidepressant

Published online May 10, 2011 treatment. Antidepressant adherence and patient satisfaction both improved in the intervention arm of the study.

Conversations about resuscitation status are challenging for doctors. Worse, they often leave patients and families agitated and confused. As Yuen et al. argue in a comprehensive review of the topic, part of the reason is that DNR discussions occur too late to allow patient participation in the process. Proposed solutions include promoting cultural change within hospitals, imposing new standards for DNR discussions, providing better training to clinicians, and creating financial incentives that encourage more comprehensive DNR discussions.

With the passage of the Affordable Care Act (ACA), health care reform is certain, but the exact form that it will take (and that will pass muster by the Supreme Court) is still unclear. The only certainty is that reform will be accompanied by acrimonious debate. We are pleased to announce that JGIM is leaping into the fray by launching a new series of Health Policy Perspectives by nationally renowned policy experts. Implementation of reform will impact the delivery system around the country for millions of Americans, and these changes will have important implications for general internists.

In the current issue, we begin with a commentary by Mark Hall, JD, one of the nation's leading scholars in health care policy and law. Mr. Hall's commentary serves as a good example of how health care reform may affect general internists, given the important role that many internists play in safety net organizations that serve those lacking adequate health insurance. In the coming months, stay tuned for more contributions tackling key aspects of health care reform. These Perspectives will explore topics as varied as the role of allied health care providers under the ACA and the impact of the ACA on Medicaid programs. One message that resounds throughout these contributions is that while it is tough to make predictions about where health care reform is going, general internists will continue to be key stakeholders in the debate.

Corresponding Author: Richard L. Kravitz, MD, MSPH; Division of General Medicine, University of California at Davis, $4150 \mathrm{~V}$. Street, Suite 2400 PSSB, Sacramento, CA 95817, USA (email: rlkravitz@ucdavis.edu). 